\title{
TRANSFORMACIÓN ESTRUCTURAL Y PRODUCTIVIDAD SECTORIAL EN COSTA RICA ${ }^{1}$
}

\author{
STRUCTURAL TRANSFORMATION AND SECTORAL PRODUCTIVITY IN COSTA RICA
}

TRANSFORMAÇÃO ESTRUTURAL E PRODUTIVIDADE SETORIAL NA COSTA RICA

\section{Alonso Alfaro Ureña ${ }^{2}$ \\ Alberto Vindas Quesada ${ }^{3}$}

\begin{abstract}
Resumen
Este trabajo documenta el proceso de transformación estructural que experimentó Costa Rica a partir de 1950 y por las siguientes seis décadas. Tal como ha sido ampliamente documentado en la literatura, los países que incrementan el ingreso de sus habitantes usualmente experimentan una reasignación de trabajo desde el sector agrícola hacia el sector manufactura y el de servicios. Para Costa Rica se observa un patrón similar que, no obstante, tiene particularidades que se discutirán a fondo. En este documento se presentan ejercicios contrafactuales que toman como punto de partida el modelo de Duarte \& Restuccia (2010), calibrado con la información actualizada para Costa Rica y Estados Unidos.

Palabras claves: productividad; transformación estructural; productividad sectorial

\section{Abstract}

This paper documents the structural transformation process in Costa Rica during the 1950s and the subsequent six decades. It is widely documented
\end{abstract}

Doi: https://doi.org/10.15359/eys.24-55.4

Fecha de recepción: 08-01-2019. Fechas de reenvíos: 19-02-2019, 12-03-2019. Aceptado el 13-03-2019. Publicado el 13-03-2019.

${ }^{1}$ Las ideas expresadas en este documento son de los autores y no necesariamente representan las del BCCR.

2 Ph.D. en Economía. Investigador del Departamento de Investigación Económica del Banco Central de Costa Rica (BCCR), Costa Rica. Correo electrónico alfaroua@bccr.fi.cr https://orcid.org/0000-0001-6241-0732

${ }^{3}$ Máster en Economía. Investigador del Departamento de Investigación Económica del Banco Central de Costa Rica (BCCR), Costa Rica. Correo electrónico avindas@bccr.fi.cr https://orcid.org/0000-0002-3607-8577

Alonso Alfaro Ureña / Alberto Vindas Quesada



Revista Economía y Sociedad by Universidad Nacional is licensed under a CreativeCommons Reconocimiento-NoComercial- 
that countries that grow in terms of income see employment shift away from the agricultural sector to the manufacturing and services sector. This same pattern can be observed in Costa Rica. However, this process has some peculiarities that will be discussed here. The paper presents counterfactual exercises using the Duarte \& Restuccia (2010) model, calibrated with Costa Rican and American data.

Keywords: productivity; structural transformation; sectoral productivity

\section{Resumo}

Este trabalho documenta o processo de transformação estrutural experimentado pela Costa Rica a partir de 1950 e pelas seis décadas seguintes. Assim como tem sido amplamente documentado na literatura, os países que aumentam a renda de seus habitantes geralmente experimentam uma realocação de trabalho do setor agrícola para o setor manufatureiro e o setor de serviços. Para a Costa Rica, existe um padrão semelhante que, no entanto, tem particularidades que serão discutidas em profundidade. Este documento apresenta exercícios contracfatuais que tomam como ponto de partida o modelo de Duarte \& Restuccia (2010), calibrado com informações atualizadas para a Costa Rica e os Estados Unidos.

Palavras-chave: produtividade; transformação estrutural; produtividade setorial

\section{Introducción}

En este trabajo se documenta la transformación estructural que ha experimentado Costa Rica en los últimos sesenta años y los cambios en la productividad de los sectores ${ }^{4}$ que componen la economía. Ambos elementos permiten explicar la evolución de la productividad agregada de la economía del país, la cual ha experimentado rezagos considerables en comparación con países desarrollados (especialmente con respecto a Estados Unidos). Los resultados de este trabajo podrían servir de guía para identificar en qué sectores de la economía existe potencial de crecimiento para la productividad agregada del país y para motivar una discusión sobre las razones de por qué ha ocurrido el estancamiento durante las últimas décadas.

\footnotetext{
${ }^{4}$ Desde el punto de vista de las cuentas nacionales se identifican los siguientes sectores institucionales: hogares, sociedades no financieras, instituciones financieras, instituciones sin fines de lucro y administración pública; no obstante, en este documento se hará referencia a sectores para identificar el grupo de actividades productivas orientadas a: agricultura, manufactura, y servicios.
}

54

Alonso Alfaro Ureña / Alberto Vindas Quesada

(c) (1) 8 (2)

Revista Economía y Sociedad by Universidad Nacional is licensed under a CreativeCommons Reconocimiento-NoComercial- 
En este documento se utilizará una métrica de productividad equivalente al valor agregado por hora trabajada ${ }^{5}$. En Costa Rica esta variable aumentó en promedio anual un 1,46 \% desde 1950 hasta 2010, mientras que para la economía estadounidense creció un 1,98 \%. Esto implica que la productividad en Estados Unidos se duplica, en promedio, en 35 años, mientras que la economía costarricense tarda 48 años en alcanzar el mismo hito. El crecimiento de la productividad es determinante fundamental del nivel de ingreso de los países, por lo que mientras el crecimiento en Costa Rica sea inferior, será imposible lograr la convergencia de los niveles de ingreso entre ambos países. Esta realidad plantea interrogantes sobre cómo cumplir el reto de que Costa Rica alcance los niveles de ingreso de un país desarrollado.

Existe, no obstante, alguna evidencia empírica alentadora. Al analizar los datos desagregados resulta claro que Costa Rica ha incrementado (aunque levemente) la productividad relativa a Estados Unidos en el sector de manufactura. La razón principal del rezago en términos agregados se debe al lento crecimiento en la productividad de servicios. No solamente ha caído la productividad de este sector, sino que también la economía costarricense ha experimentado una expansión considerable de este mismo sector. Este es el mismo proceso de transformación estructural que enfrentan las economías al desarrollarse, pero que amplifica la caída de manera relativa para la economía.

Una forma de evaluar la importancia de cada sector en la evolución de la productividad agregada del país es utilizar un modelo que replique los hechos estilizados de esta transformación estructural que ha experimentado el país. Por esta razón, se presenta un modelo de equilibrio general propuesto por Duarte \& Restuccia (2010) que puede ser calibrado para una economía en particular y que permite realizar ejercicios contrafactuales tomando en cuenta diferentes trayectorias para la productividad de cada sector. El modelo consiste de tres sectores productivos (agricultura, manufactura y servicios) y replica, de manera razonable, los cambios en la distribución de horas trabajadas en la economía ante cambios en la productividad de cada sector y el ingreso disponible de los hogares.

Este estudio se enmarca en una creciente literatura que estudia el crecimiento económico, transformación estructural y productividad en Costa Rica. OCDE (2018) presenta en el primer capítulo un diagnóstico completo sobre la evolución de la productividad en los últimos años para Costa Rica. El último capítulo discute cómo la mala asignación de los recursos podría estar causando un rezago en productividad con respecto a los países desarrollados.

\footnotetext{
${ }^{5}$ Si se utilizara una medición de productividad total de factores (PTF) las conclusiones a las que se podrían llegar con respecto a la variación desagregada por sector son similares. Sin embargo, el crecimiento de $Y / L$ en agregado puede estar relacionado con variaciones en la PTF, en el capital por producto $(K / Y)$, o en el capital humano $(h)$. Ver Klenow \& Rodríguez-Clare (1997).
}

Alonso Alfaro Ureña / Alberto Vindas Quesada

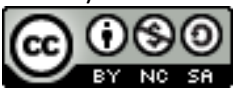

Revista Economía y Sociedad by Universidad Nacional is licensed under a CreativeCommons Reconocimiento-NoComercial- 
Abarca y Ramírez (2016) discuten que "desde las reformas post-crisis de los ochenta, sin embargo, la acumulación de trabajo y capital han sido los factores de producción que han explicado el crecimiento de la economía". Estos resultados contrastan con los presentados en Robles (2018), quien documenta el incremento en productividad durante los últimos años. También Mulder, Patiño-Pascumal y Monge-González (2016) documentan un aumento de productividad entre los años 2001 y 2015, pero cuyo crecimiento fue menor debido al cambio del empleo hacia sectores con menores niveles y menores tasas de crecimiento en productividad. Este resultado es cualitativamente similar al que se deduce del modelo presentado en el trabajo actual.

En este documento se discutirá el contexto histórico de la transformación estructural de Costa Rica y se describirá la evolución de la productividad en los diferentes sectores de la economía. Se discutirán brevemente las razones de dichos cambios, los cuales contrastan significativamente con los que enfrentó una economía como la estadounidense tanto por el nivel de desarrollo en el que se encontraba en 1950 como por los diferentes ciclos económicos que ha experimentado. Adicionalmente, Costa Rica ha realizado cambios en política económica muy distintos a los de Estados Unidos en el periodo analizado. Se discutirá, también, brevemente, cómo se construyeron los datos requeridos para este trabajo.

Las siguientes secciones consisten en el modelo y las calibraciones alternativas que se proponen: una para la economía estadounidense (con un ajuste para que pueda replicar el proceso de transformación costarricense), y otra para la economía costarricense. El primer ejercicio de calibración permite realizar comparaciones de productividad entre ambas economías, mientras que el segundo ejercicio permite identificar diferencias entre las economías (manifestadas en los parámetros de calibración), y facilita la realización de ejercicios contrafactuales de cambios en la productividad.

\section{Caracterización histórica y datos}

\section{Caracterización histórica}

Costa Rica es una economía pequeña y abierta que ha experimentado el mismo proceso de transformación estructural que se observa en la mayoría de economías en desarrollo. Al analizar la composición del empleo ${ }^{6}$ durante las últimas seis décadas es posible identificar los patrones usuales en los que hay una caída sostenida en la proporción de horas dedicadas a agricultura, un aumento constante en las horas dedicadas a servicios, y un aumento temporal en las horas en manufactura, en el que posteriormente empiezan a caer. La Figura 1 presenta estos datos para Costa Rica.

\footnotetext{
${ }^{6}$ En este trabajo se utiliza como medida del empleo de la economía y los sectores que la componen el número de horas trabajadas, por lo que ambos términos se utilizaran indistintamente.

56

Alonso Alfaro Ureña / Alberto Vindas Quesada

(c) (†) 8 ()

Revista Economía y Sociedad by Universidad Nacional is licensed under a CreativeCommons Reconocimiento-NoComercial- 
El año en el que el sector de manufactura alcanzó la máxima proporción en la composición del empleo fue en 1991, cuando un 31,7 \% de las horas estuvieron dedicadas a este sector. Uno de los elementos más relevantes que se pueden apreciar en los datos es el efecto de la crisis económica de inicios de los años ochenta. Durante esta década hubo un leve descenso en la participación de manufactura en el total de horas trabajadas. Un fenómeno similar está presente en los servicios, cuya proporción del empleo dejó de crecer al ritmo que lo hacía en las décadas previas. Consecuentemente, el sector agrícola absorbió temporalmente más sujetos, por lo que el descenso en la proporción de empleo de este sector se detuvo considerablemente.

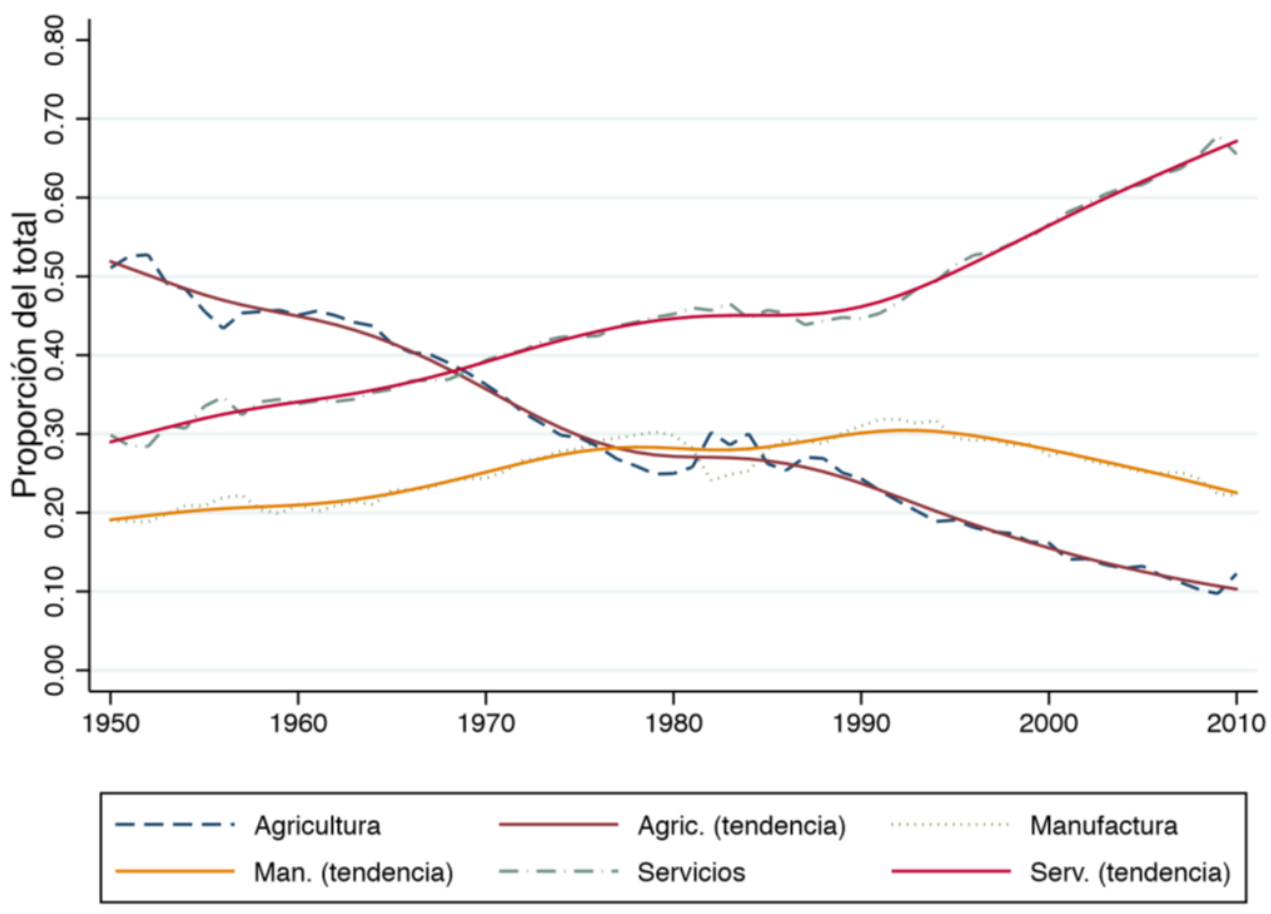

Figura 1. Costa Rica: Proporción de horas trabajadas por sector. Fuente: elaboración propia con base en datos de Penn World Table (PWT) versión 8.0 (ver Heston, Summers y Aten, 2012) y , Feenstra \& Timmer (2013), Total Economy Database (TED), ver The Conference Board (2014), la 10 Sector Database, Timmer, de Vries \& de Vries (2014), y el INEC.

Estos cambios en las tendencias ocurridos a partir de la crisis de la década de los ochenta se pueden interpretar como una respuesta ante la caída del ingreso que ocurrió en los primeros años de la crisis. El consumo de bienes de manufactura y especialmente de servicios está negativamente relacionado al nivel de ingreso de los países, por lo que un choque negativo tal como el ocurrido haría que el sector agrícola aumente o, al menos, mantenga la importancia relativa en cuanto a la proporción del empleo. Adicionalmente, entre los ochenta y mitad de los 
noventa hay parte del crecimiento del empleo en la actividad agrícola debido a la estrategia de promoción de las exportaciones no tradicionales. Una vez que la economía costarricense comenzó a crecer de nuevo, el proceso de transformación estructural continua tal y como ocurría previo a la crisis.

De manera simultánea a cambios en el ingreso hubo cambios en la productividad de cada uno de los sectores que, de la misma manera, tienen consecuencias sobre la composición del empleo en la economía. La Figura $\underline{2}$ presenta la evolución del valor agregado por hora de Costa Rica en las últimas seis décadas mientras que la Figura $\underline{3}$ presenta los datos correspondientes para la economía estadounidense.

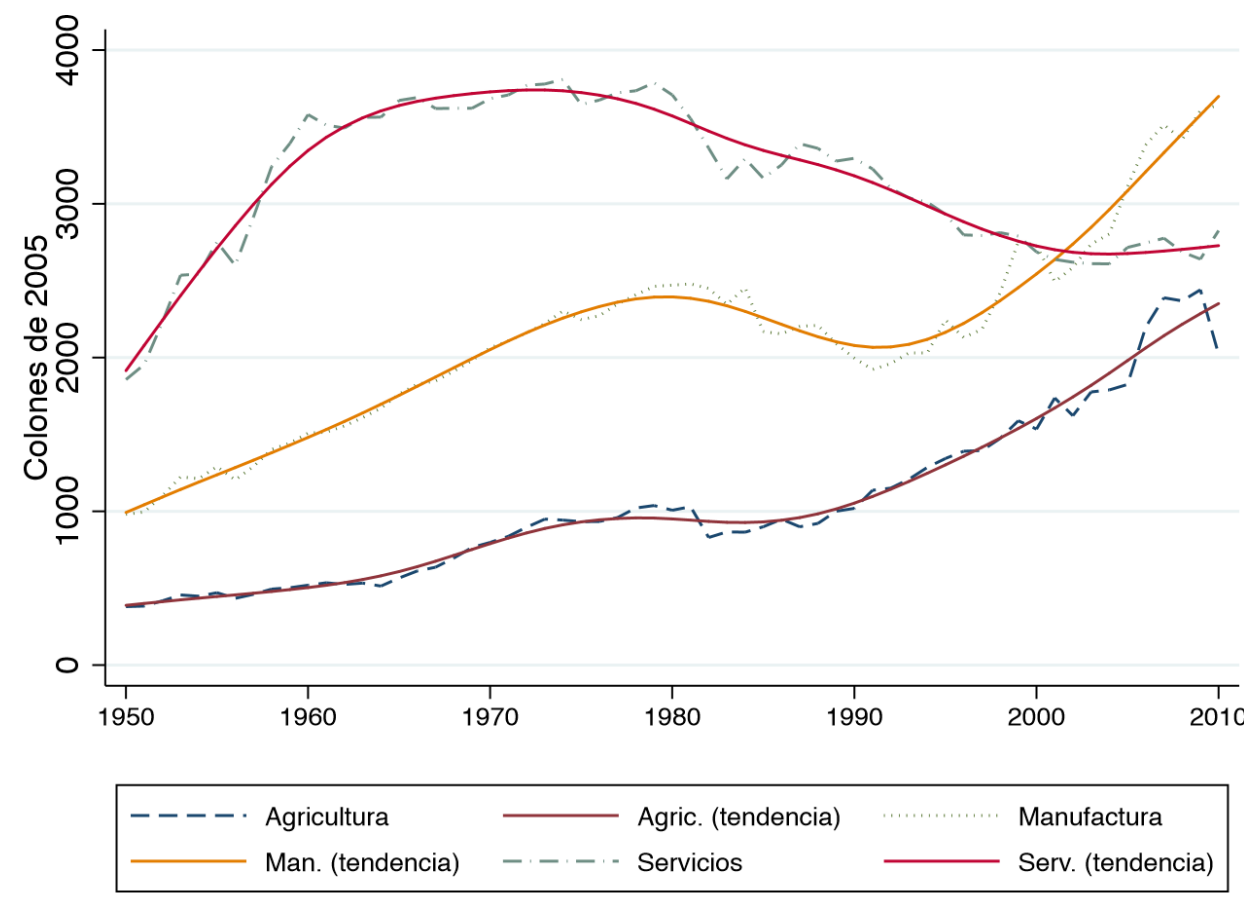

Figura 2. Costa Rica: Valor agregado por hora. Fuente: elaboración propia con base en datos de Penn World Table (PWT) versión 8.0, Feenstra \& Timmer (2013), Total Economy Database (TED), ver The Conference Board (2014), la 10 Sector Database, Timmer et al. (2014), y el INEC.

58 


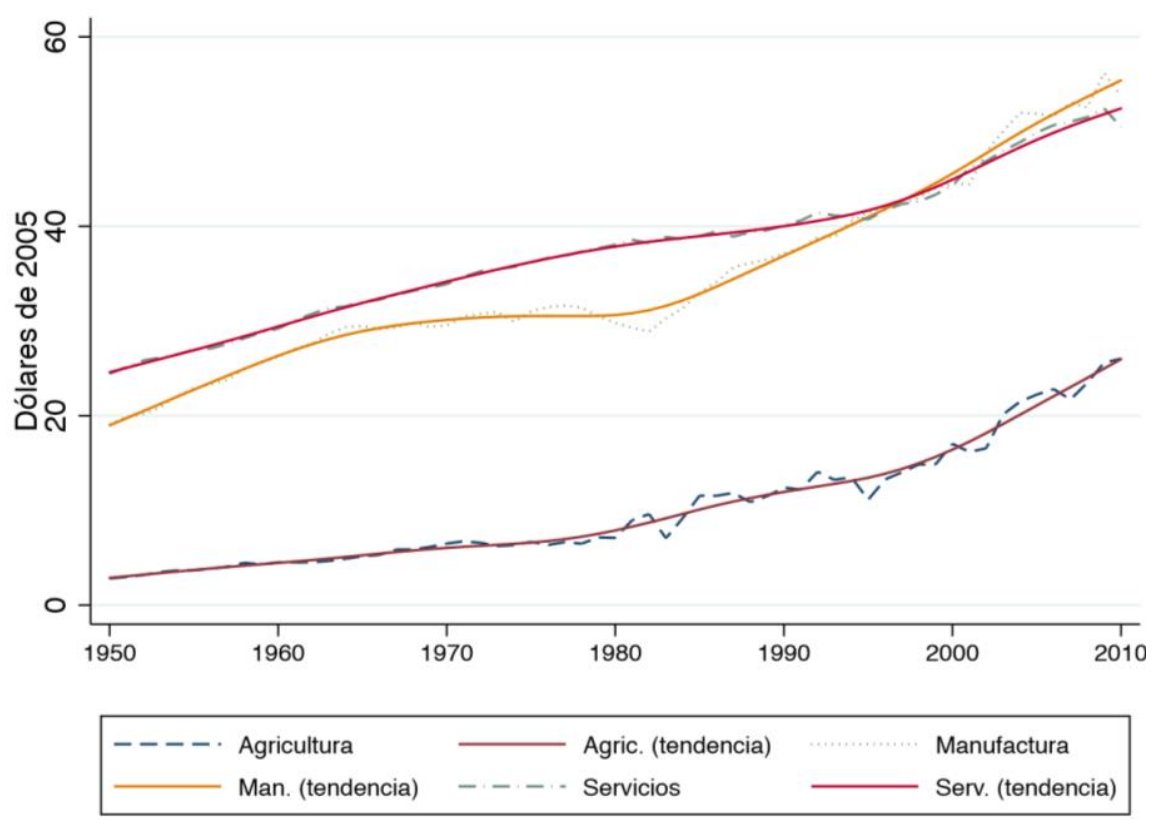

Figura 3. Estados Unidos: Valor agregado por hora. Fuente: elaboración propia con base en datos de Penn World Table (PWT) versión 8.0, Feenstra \& Timmer (2013), Total Economy Database (TED), The Conference Board (2014), la 10 Sector Database, Timmer et al. (2014), y el INEC.

Las diferencias en la evolución de la productividad de ambos países son considerables. En el sector manufacturero de Costa Rica hay una caída en la productividad en la década de los ochenta, la cual vuelve a crecer solamente a inicios de la siguiente década. Por el contrario, en Estados Unidos este es uno de los periodos en los que la productividad del sector creció más rápidamente.

La mayor diferencia radica en que hay patrones totalmente diferentes para la productividad en agricultura y servicios para los dos países. Mientras la productividad en servicios ha crecido en EE. UU. de manera constante durante más de medio siglo, en Costa Rica hubo una reversión de la tendencia de crecimiento de la productividad desde inicio de los años setenta, la cual continuó cayendo durante las siguientes tres décadas. La caída en la productividad por hora de este sector solamente se ha revertido (aunque muy modestamente) en la última década.

Esta particularidad reviste una importancia considerable en el análisis de la evolución de la productividad de la economía costarricense. El único sector cuyo valor agregado por hora ha caído durante las últimas décadas es el que ha absorbido la mayor cantidad de trabajo en la economía, alcanzando una importancia relativa de alrededor de 70 \%. Esta es la razón más importante para plantear y calibrar un modelo que permita replicar el comportamiento de la economía durante 
las últimas décadas. Es necesario comprender cuáles son las fuerzas que han impulsado la expansión de este sector a pesar de que, en apariencia, no ha sido exitoso en incrementar su productividad.

En cuanto a la productividad del sector agrícola, la diferencia radica en la temporalidad de los incrementos. En la primera mitad del periodo de estudio hay un crecimiento muy lento del valor agregado por hora de Costa Rica en comparación con el de los EE.UU. En la segunda parte del periodo el crecimiento del sector se acelera en Costa Rica, aunque no tanto como el de su contraparte.

La Figura $\underline{4}$ resume estas características históricas de la evolución de la productividad de Costa Rica con respecto a Estados Unidos. Esta es una figura que presenta los resultados del modelo y la correspondiente calibración para la economía costarricense con respecto a la estadounidense, que se presentarán en las secciones 3 y $4^{7}$. Sin embargo, resulta conveniente hacer referencia a estos resultados en esta contextualización histórica. Los resultados indican que la década de los sesentas fue un periodo de tiempo donde el incremento en productividad relativa fue impulsado por el sector servicios, mientras el impulso en el crecimiento de la productividad relativa en los setentas fue facilitado, más bien, por el sector de manufactura.

Estos patrones son similares a los observados en Rodríguez-Clare, Sáenz \& Trejos (2003) y corresponden a periodos específicos de políticas económicas de desarrollo. Identifican el periodo comprendido entre 1950 y 1963 como el periodo de Transición del modelo agroexportador al modelo de sustitución de importaciones. En los datos de Costa Rica presentados previamente es evidente un incremento sustancial en el valor agregado por hora del sector servicios, que es muy posiblemente una consecuencia directa del crecimiento en la inversión pública en los servicios durante las décadas de los cuarenta y cincuenta, especialmente en educación, salud, y el sector financiero.

Los mismos autores identifican el periodo comprendido entre 1973 y 1980 como el de Estado empresario y política macroeconómica insostenible. En este periodo se crearon una gran cantidad de empresas publicas mediante la Corporación de Desarrollo S.A. (CODESA), el cual se manifiesta en los datos como un incremento en la productividad del sector de manufactura, debido primordialmente a una mayor cantidad de obras de infraestructura.

\footnotetext{
${ }^{7}$ Estos resultados no son directamente obtenidos de los datos presentados en las Figuras $\underline{2}$ y $\underline{3}$ debido a que las productividades sectoriales no están medidas en unidades comparables entre países. No existen mediciones históricas de datos de producción en paridad poder de compra (PPC). En la siguiente sección se discutirá cuál es la estrategia que se debe seguir para obtener datos de productividad comparables entre países.
}

60

Alonso Alfaro Ureña / Alberto Vindas Quesada

(c) (†) 8 ()

Revista Economía y Sociedad by Universidad Nacional is licensed under a CreativeCommons Reconocimiento-NoComercial- 


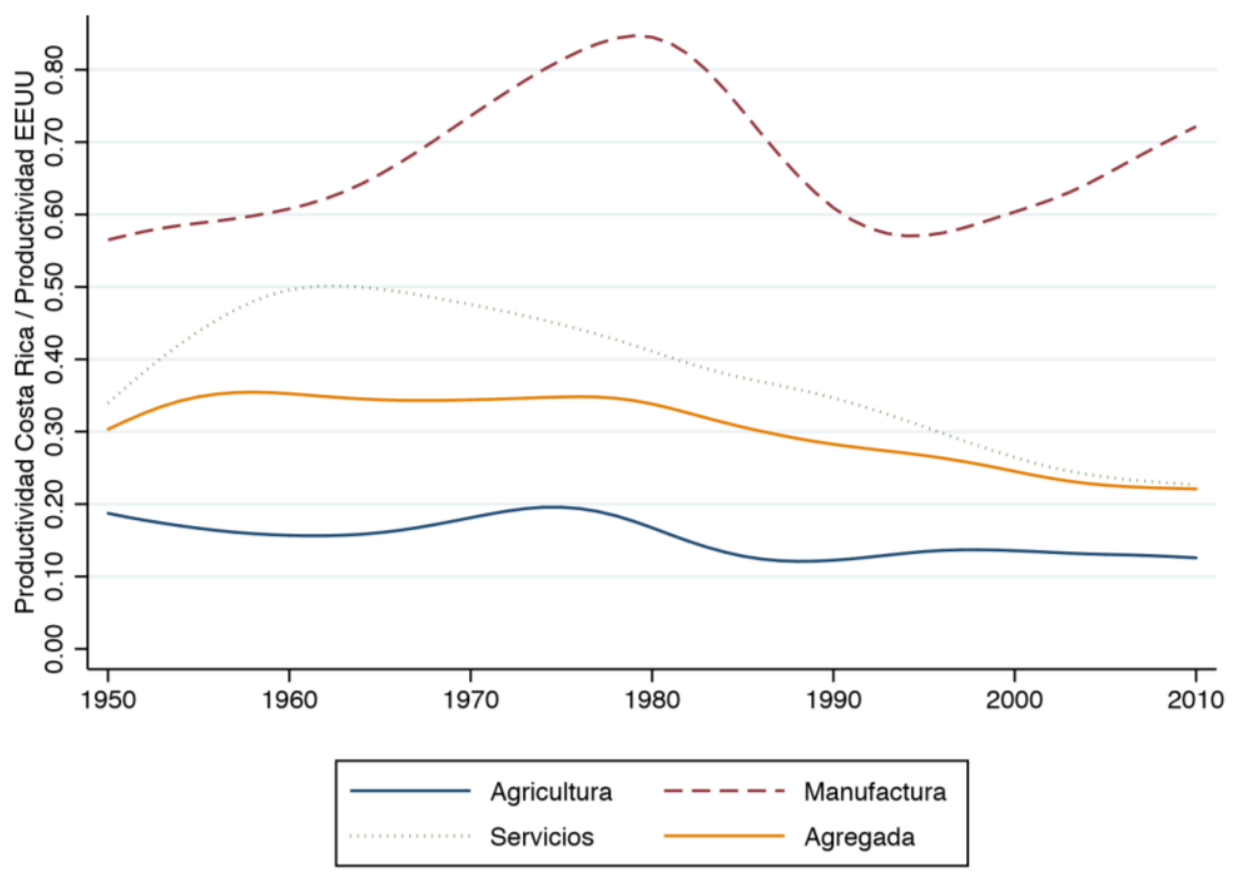

Figura 4. Productividades de Costa Rica relativas a Estados Unidos (Resultados del modelo presentado en la Sección 3). Fuente: elaboración propia con base en datos de Penn World Table (PWT) versión 8.0, Feenstra \& Timmer (2013), Total Economy Database (TED), The Conference Board (2014), la 10 Sector Database, Timmer et al. (2014), y el INEC.

Una diferencia considerable entre los dos países es que la productividad de los sectores de manufactura y servicios en Estados Unidos sigue tendencias muy similares, y duplican la productividad del sector agrícola. En el período posterior a la década de los noventas, esto se puede atribuir principalmente a la presencia y crecimiento de inversión extranjera directa (IED). Para Costa Rica la productividad de servicios y de agricultura son considerablemente más bajas a la de manufactura. En ambos sectores hay una actividad productiva dinámica integrada al comercio internacional, mientras que hay otro tipo de actividad que no está sujeta a competencia por proteccionismo comercial (sector agrícola) o por la actividad del Estado (servicios). Nuevamente el caso de la productividad de servicios es llamativo, ya que el valor agregado por hora cayó, a inicios de la primera década del siglo XXI, por debajo del de manufactura.

En términos agregados, la ligera ganancia en productividad con respecto a Estados Unidos que se presenta en las primeras décadas de análisis se revirtió definitivamente en la década de los ochentas. Ha habido alguna desaceleración en la caída gracias a un crecimiento mayor que el estadounidense en la productividad del sector de manufactura. Adicionalmente, ha habido un leve crecimiento en el valor agregado por hora del sector servicios y del sector agrícola durante 
la última década, pero no ha sido suficiente para revertir la tendencia en la productividad agregada relativa. No existen suficientes señales alentadoras con respecto a una mejora relativa en el mediano plazo de la productividad del país.

\section{Datos}

Los datos requeridos para utilizar el modelo que se presentará a continuación son: 1) La proporción de horas trabajadas en cada sector. 2) El valor agregado por hora en cada sector. 3) La productividad agregada por hora. La base de datos de Duarte \& Restuccia $(\underline{2010})^{8}$ recurre a algunos supuestos para las magnitudes de horas trabajadas para Costa Rica, los cuales se revisaron para incorporar algunas mejoras en la construcción de las series históricas con información adicional. Para efectos de comparabilidad, se utilizan las mismas fuentes tanto para Costa Rica como para Estados Unidos, cuando es posible ${ }^{9}$.

Los datos agregados, PIB per cápita y número total de horas trabajadas, se extrajeron de la Penn World Table (PWT) versión 8.0, Feenstra \& Timmer (2013), y la Total Economy Database (TED), The Conference Board (2014), respectivamente. Para la construcción de las horas trabajadas es requerido utilizar algunos supuestos, los cuales se detallan en el Apéndice $A$.

Los datos sectoriales de valor agregado por hora se obtienen, para ambos países, de la 10 Sector Database, Timmer et al. (2014). En esta base de datos existe información del empleo por cada uno de los sectores, pero no hay información con respecto al número de horas trabajadas. Sin embargo, fue posible construir una base de datos de horas trabajadas utilizando información de KLEMS para EE. UU. (ver O'Mahony y Timmer, 2009) y del Instituto Nacional de Estadística y Censos (INEC) para Costa Rica. En el caso costarricense, tal como encontraron de manera general Duarte \& Restuccia (2010), las razones de horas trabajadas en cada uno de los sectores con respecto a las horas trabajadas del total de la economía son sumamente estables para el período 1990-2010, datos que se suponen constantes para el resto del período. Los detalles con respecto a la construcción de estas series también se incluyen en el Apéndice A. Todas las variables utilizadas se filtran utilizando Hodrick-Prescott con parámetro $\lambda=100$.

\section{Modelo}

La evidencia empírica presentada en la sección anterior muestra un proceso de transformación estructural en la economía costarricense que no ha sido ocasionado exclusivamente por incrementos en la productividad de los sectores que aumentan su participación relativa en la economía. El sector servicios es el único sector que ha reducido el valor agregado por hora. No

\footnotetext{
${ }^{8}$ Bases de datos disponibles en http://www.economics.utoronto.ca/diegor/research/research.html

${ }^{9}$ Esto implica que algunos datos para los Estados Unidos se obtuvieron de fuentes diferentes a la base de datos original.
}

62

Alonso Alfaro Ureña / Alberto Vindas Quesada

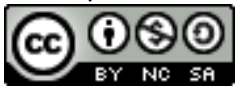

Revista Economía y Sociedad by Universidad Nacional is licensed under a CreativeCommons Reconocimiento-NoComercial- 
obstante, es el sector que ha crecido de manera más drástica en importancia en el número de horas de trabajo. De la misma manera, el sector manufacturero es el único que ha incrementado su propia productividad en términos absolutos y en comparación con el mismo sector en los Estados Unidos, pero es un sector que ha visto contraída su participación del empleo total.

El objetivo de esta sección es introducir un modelo que permita replicar los hechos estilizados presentados. El modelo necesariamente tiene que permitir diferencias en productividad de los sectores, pero también preferencias no homotéticas de manera que algunos sectores crezcan en importancia por un efecto ingreso de la economía. El modelo calibrado permitirá realizar ejercicios contrafactuales para evaluar cuáles son los elementos determinantes del crecimiento de la productividad agregada de la economía.

El modelo que se resume a continuación es el presentado por Duarte \& Restuccia (2010), el cual se fundamenta en Rogerson (2008). Dadas las productividades de un país el modelo encuentra la asignación de trabajo en equilibrio para los tres sectores de la economía. El modelo está construido de manera que replica satisfactoriamente el proceso de transformación estructural que atraviesan las economías al incrementar el nivel de ingreso y al enfrentar diferentes niveles de productividad en cada sector.

Ngai \& Pissarides (2007) describen que en la literatura reciente se presentan usualmente dos explicaciones para la transformación estructural de las economías: una se fundamenta en preferencias no homotéticas que tienen como consecuencia elasticidades-ingreso distintas entre los bienes de consumo. La segunda explicación se basa en cambios tecnológicos, lo que implica que hay distintas tasas de crecimiento de la productividad en cada uno de los sectores. El modelo desarrollado por Rogerson (2008) combina ambas ideas, pues incluye preferencias no homotéticas que son centrales en la explicación de la disminución de las horas trabajadas en agricultura. Simultáneamente hay diferencias en las productividades que ayudan a explicar el crecimiento en las horas asignadas al sector servicios.

En resumen, hay dos mecanismos que operan en este modelo que permiten replicar los hechos estilizados. En primer término, el modelo se construye para que necesariamente un incremento de los ingresos ocasione que haya una menor demanda relativa de bienes agrícolas. Un nivel de ingresos muy bajo implica que toda la producción se tiene que dedicar a satisfacer las necesidades básicas de alimentación de los individuos. Una vez que el ingreso incrementa, la importancia relativa de este sector disminuye. El caso contrario ocurre con el sector servicios, ya que las preferencias se construyen para que este consumo incremente ante ingresos altos. El otro elemento que caracteriza el modelo es que hay una reasignación del empleo dependiendo de la productividad del sector: un sector con una productividad muy alta atraerá grupos trabajadores, ya que hay una potencial ganancia salarial (valor del producto marginal). 
La economía modelo se caracteriza por tres sectores productivos: agricultura, manufactura y servicios. La producción de cada uno de estos sectores utiliza una tecnología de rendimientos constantes a escala:

$$
Y_{i}=A_{i} L_{i}, \quad i \in\{a, m, s\}
$$

donde $Y_{i}, A_{i}$ y $L_{i}$ representan la producción total en el sector $i$, la productividad por hora de ese sector y las horas totales trabajadas. Se supone también la existencia de un continuo de empresas que son perfectamente competitivas en el mercado de factores. Por lo tanto, dados los precios de los productos y salarios, el problema de la empresa representativa es:

$$
\text { máx } L_{L_{i} \geq 0}\left\{p_{i} A_{i} L_{i}-w L_{i}\right\}
$$

La economía está poblada por hogares representativos con vida infinita, de tamaño constante y masa unitaria. Cada hogar está dotado de una cantidad de horas $L$, las cuales ofrece inelásticamente al mercado. Las preferencias se representan de la siguiente manera:

$$
\sum_{t=0}^{\infty} \beta^{t} u\left(c_{a, t}, c_{t}\right), \quad \beta \in(0,1)
$$

donde $c_{a, t}$ es el consumo de bienes agrícolas en el periodo $t$ y $c_{t}$ es el consumo de un bien compuesto de bienes de manufactura y servicios. La función de utilidad instantánea viene dada por una de tipo Stone-Geary:

$$
u\left(c_{a, t}, c_{t}\right)=a \log \left(c_{a, t}-\bar{a}\right)+(1-a) \log \left(c_{t}\right), \quad a \in(0,1)
$$

donde $\bar{a}>0$ puede interpretarse como un nivel de subsistencia de bienes agrícolas, por debajo del cual el hogar no puede sobrevivir. Por encima de este nivel de subsistencia, la elección entre los bienes agrícolas y los bienes compuestos tiene elasticidad de sustitución constante. El bien compuesto no agrícola está dado por:

$$
c_{t}=\left[b c_{m, t}^{\rho}+(1-b)\left(\left(c_{s, t}+\bar{s}\right)^{\rho}\right]^{1 / \rho}\right.
$$

donde $c_{m, t}$ representa el consumo del bien manufactura y $c_{s, t}$ el consumo de servicios. Además $\bar{s}$ $>0$, el cual podría interpretarse como el consumo constante de bienes de servicio en el hogar. Finalmente, $b \in(0,1)$ y $\rho<1$. En este modelo no hay decisiones intertemporales, por lo que el problema de los hogares es estático en cada momento del tiempo:

$$
\text { máx }{ }_{C_{i} \geq 0}\left\{a \log \left(c_{a}-\bar{a}\right)+\frac{1-a}{\rho} \log \left[b c_{m, t}^{\rho}+(1-b)\left(\left(c_{s, t}+\bar{s}\right)^{\rho}\right]\right\}\right.
$$

sujeto a:

64

Alonso Alfaro Ureña / Alberto Vindas Quesada

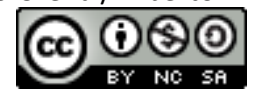

Revista Economía y Sociedad by Universidad Nacional is licensed under a CreativeCommons Reconocimiento-NoComercial- 


$$
p_{a} c_{a}+p_{m} c_{m}+p_{s} c_{s}=w L
$$

donde $p_{i}$ denota el precio de cada uno de los bienes $i$.

El equilibrio en los mercados de trabajo y de bienes requiere que las siguientes restricciones se cumplan en cada periodo:

$$
\begin{array}{r}
L_{a}+L_{m}+L_{s}=L \quad(3.4) \\
c_{a}=Y_{a} \quad c_{m}=Y_{m} \quad c_{s}=Y_{s}
\end{array}
$$

Un equilibrio competitivo es un conjunto de precios $\left\{p_{a}, p_{m}, p_{s}\right\}$, un conjunto de asignaciones de consumo $\left\{c_{a}, c_{m}, c_{s}\right\}$ y un conjunto de asignaciones de trabajo $\left\{L_{a}, L_{m}, L_{s}\right\}$ para los cuales las empresas resuelven su problema de maximización de beneficios dado en (3.2), los hogares resuelven su problema de maximización de utilidad dado en (3.3), y los mercados se encuentran en equilibrio según (3.4) y (3.5).

Las condiciones de primer orden para la empresa requieren que el costo marginal, representado por los salarios por unidad de producción, sea igual al ingreso marginal, representado por el precio del bien:

$$
p_{i}=\frac{w}{A_{i}}
$$

Para encontrar el equilibrio del modelo se normaliza el salario igual a uno. Las condiciones de primer orden para la elección entre el consumo agrícola y el del bien compuesto se resumen en:

$$
L_{a}=(1-a) \frac{\bar{a}}{A_{a}}+a\left(L+\frac{\bar{s}}{A_{s}}\right)
$$

Cuando $a>0$ un crecimiento de las productividades positivo implica que la proporción de horas dedicadas a la producción agrícola $L_{a} / L$ tiende a $a$, por lo que este parámetro se interpreta como la proporción de horas asignadas a agricultura de largo plazo. ${ }^{10}$.

Las condiciones de primer orden para la elección entre el consumo de bienes de manufactura y servicios implican que:

$$
\frac{b}{1-b}\left(\frac{c_{m}}{c_{s}+\bar{s}}\right)^{\rho-1}=\frac{p_{m}}{p_{s}}
$$

${ }^{10}$ Esta expresión evidencia la diferencia principal entre el modelo de Duarte \& Restuccia (2010), y los que se presentan en Rogerson (2008) y Duarte \& Restuccia (2007). En los últimos se presenta una función de utilidad $u\left(c, c_{a}\right)$ $=U(c)+V\left(c_{a}\right) \operatorname{con} V\left(c_{a}\right)=\min \left\{c_{a}, a^{-}\right\}$, lo que implica que la producción y el consumo per cápita de bienes agrícolas sea constante. Este sería el caso si $a=0$.

Alonso Alfaro Ureña / Alberto Vindas Quesada

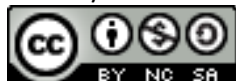

Revista Economía y Sociedad by Universidad Nacional is licensed under a CreativeCommons Reconocimiento-NoComercial- 
Haciendo uso del equilibrio en el mercado de trabajo y las condiciones de primer orden para la empresa, se puede reescribir:

$$
L_{m}=\frac{L-L_{a}+\bar{s} / A_{s}}{1+x}
$$

donde

$$
x \equiv\left(\frac{b}{1-b}\right)^{1 /(1-\rho)}\left(\frac{A_{m}}{A_{s}}\right)^{\rho /(1-\rho)}
$$

En estas últimas dos expresiones se puede ilustrar el mecanismo según el cual se da la reasignación de horas de trabajo. Para cualquier tipo de preferencias, pero manteniendo la productividad relativa entre sectores constante, la variable $x$ se mantendría invariable. Sin embargo, en el numerador de (3.8), el término $\bar{s} / A_{s}$ decrecería siempre que aumente la productividad del sector de servicios, lo que a la vez aumentaría la cantidad de trabajo asignada al sector manufactura y llevando a la razón $L_{m} / L_{s}$ a tender a $1 / x$.

El segundo mecanismo, atribuible a las diferencias en las productividades relativas, opera a través de (3.9). Como se mencionó, en el largo plazo la razón $L_{m} / L_{s}$ tiende a $1 / x$, pero cuando las productividades relativas cambian, estas afectan (3.9). Para que haya reasignación de trabajo entre sectores se necesita que $\rho \neq 0$. Adicionalmente, para que al aumentar la productividad en manufactura en relación con la de servicios ocurra una reasignación de trabajo hacia el sector servicios, es necesario que $\rho<0$. Cuanto más negativo sea este parámetro, la reasignación de trabajo es más elástica con respecto a cambios en la productividad.

\section{Calibración y ajuste del modelo}

En esta sección se presentarán dos estrategias de calibración diferentes para los cinco parámetros que se utilizan en este modelo. La estrategia seguida por Duarte \& Restuccia (2010) es realizar esta calibración para la economía estadounidense para el periodo 1956-2004. Al igual que Rogerson (2008), el procedimiento que siguen es normalizar las productividades $A_{i}=1$ en el primer periodo, y usar tasas de crecimiento para completar el resto de las series. Posteriormente seleccionan los parámetros que hacen que el modelo, que utiliza los datos de productividad, replique de mejor manera las asignaciones de trabajo de los datos. Al normalizar $L=1$, las asignaciones de trabajo $L_{i}$ se pueden interpretar directamente como las proporciones de horas asignadas a cada sector productivo.

Una vez que el modelo está calibrado para la economía estadounidense, es posible utilizar el modelo y el primer año de los datos para obtener productividades de cada sector de la economía costarricense relativas a la economía estadounidense. Esto es necesario debido a que no existe información sectorial comparable (ajustada por paridad poder de compra) que permita alimentar el modelo sin correr el riesgo de obviar diferencias en los niveles de precios de cada sector. Esta estrategia permite realizar comparaciones de productividad entre los dos países.

66

Alonso Alfaro Ureña / Alberto Vindas Quesada

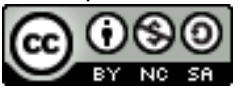

Revista Economía y Sociedad by Universidad Nacional is licensed under a CreativeCommons Reconocimiento-NoComercial- 
La otra estrategia posible es utilizar solamente la economía costarricense y calibrar el modelo. Esto permitiría realizar ejercicios contrafactuales de la evolución de la asignación de empleo entre sectores y el crecimiento de la productividad, pero no permite realizar comparaciones entre los dos países. En ambos casos, la calibración consiste en seleccionar los parámetros $a, b, \rho, \bar{a}$ y $\bar{s}$. El parámetro a representa la proporción de horas dedicadas a agricultura en el largo plazo, por lo que Duarte \& Restuccia (2010) seleccionan un valor de $1 \%(a=0,01)$ que proviene de la tendencia de largo plazo en los datos de cuentas nacionales estadounidenses.

En cualquiera de los dos casos, el procedimiento que se sigue es utilizar los datos del año inicial para la proporción de horas trabajadas en agricultura y manufactura. Estas proporciones permiten deducir valores para $\bar{a}$ y $\bar{s}$. Posteriormente, se seleccionan los valores de $b$ y $\rho$ que permitan recrear con mayor precisión la trayectoria de la proporción de horas trabajadas en manufactura y la tasa de crecimiento promedio de la productividad agregada. Esta última está dada por

$$
\begin{gathered}
\frac{Y}{L}=\sum_{i \in\{a, m, s\}} \frac{Y_{i}}{L_{i}} \frac{L_{i}}{L} \\
=\sum_{i \in\{a, m, s\}} \frac{Y_{i}}{L_{i}} \frac{L_{i}}{L}
\end{gathered}
$$

\section{Calibración para Estados Unidos}

Para Estados Unidos, el crecimiento promedio en la productividad es de casi $2 \%$, y los valores que usan en Duarte \& Restuccia (2010) $\bar{a}=0,11, \bar{s}=0,89, b=0,04$, y $\rho=-1,5$. Al modificar algunas fuentes de información y utilizar un año base diferente, es necesario recalibrar este modelo para la economía estadounidense. Los parámetros obtenidos con esta nueva calibración son $\bar{a}=$ $0,0941, s^{-}=0,8314, b=0,0263$, y $\rho=-1,4869$, los cuales son de magnitudes similares a los de referencia. Esta calibración se aprecia en la Figura $\underline{5}$. 


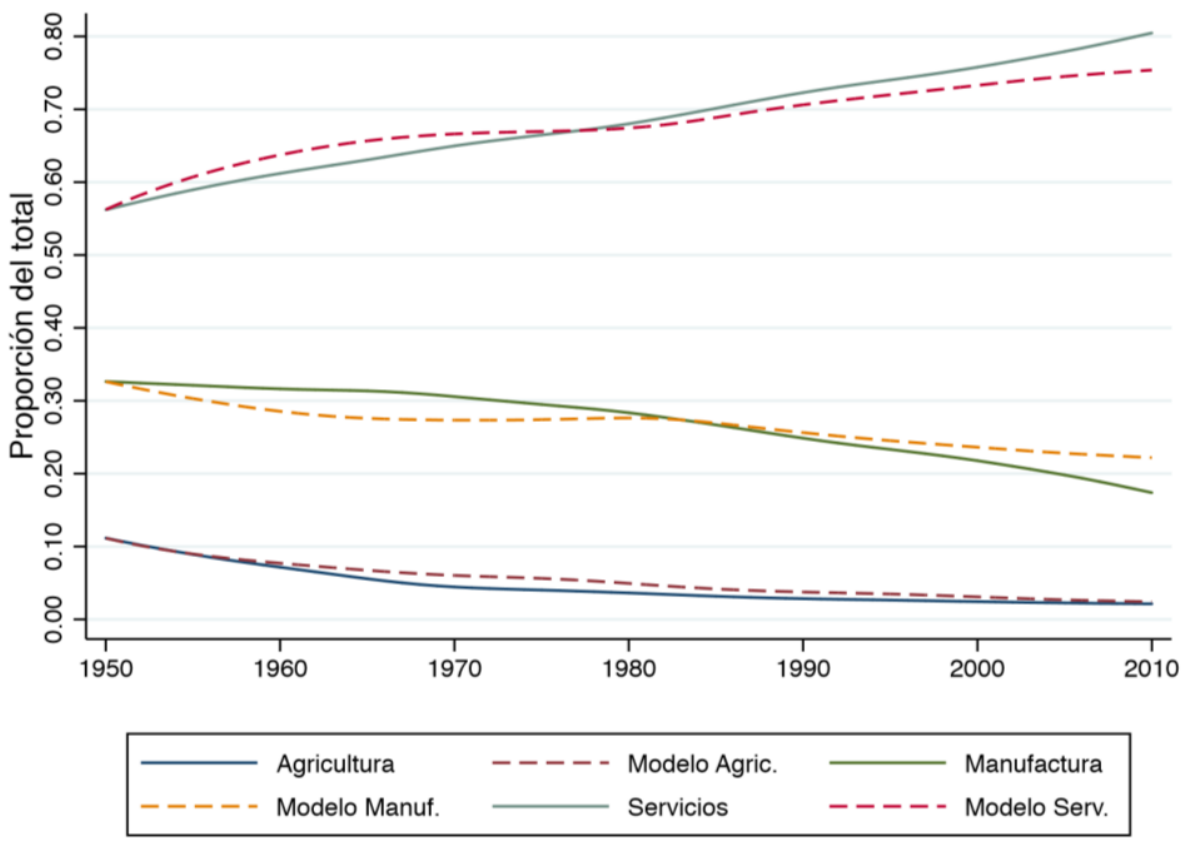

Figura 5. Modelo calibrado para Estados Unidos 1950-2010. Fuente: elaboración propia con base en datos de Penn World Table (PWT) versión 8.0, Feenstra \& Timmer (2013), Total Economía Database (TED), The Conference Board (2014), la 10 Sector Database, Timmer et al. (2014), y el INEC.

Tal como se mencionó, para realizar ejercicios de comparación con otras economías es necesario calibrar también las productividades iniciales $A_{i}$ para que las proporciones de trabajo y la productividad agregada coincidan con las del primer año de datos. En el caso de Costa Rica, esta calibración da como resultado los siguientes parámetros: $A_{a, 1950}=0,1859, A_{m, 1950}=0,5438$, y $A_{s, 1950}$ $=0,3560$. Adicionalmente, se supone que $s / A^{-}{ }_{s, 1950}=0,8314$ para cada país ${ }^{11}$. El resto de la serie se completa utilizando el crecimiento real de la productividad en cada sector en los datos. De la misma manera, el modelo produce una asignación sectorial de las horas trabajadas.

En la Figura $\underline{6}$ se aprecia que la calibración de este modelo no es satisfactoria en replicar el comportamiento de la economía costarricense, pues sobreestima la participación que debería tener el sector servicios en el total de horas trabajadas. La razón principal para que el modelo espere este comportamiento es la baja productividad del sector agrícola, la que tendería a expulsar a personas trabajadoras hacia el sector cuya productividad es mayor (en particular durante las primeras tres décadas de análisis). La contraparte de esto es que el modelo subestima la participación en el sector agrícola y en el de manufactura.

\footnotetext{
${ }^{11}$ El razonamiento detrás de este ajuste es que $\bar{S}$ debe variar con cambios grandes en la productividad de servicios. Esto por cuanto este parámetro se puede interpretar como los servicios producidos en el hogar.
}

68

Alonso Alfaro Ureña / Alberto Vindas Quesada

(c) (i) 8 (2)

Revista Economía y Sociedad by Universidad Nacional is licensed under a CreativeCommons Reconocimiento-NoComercial- 


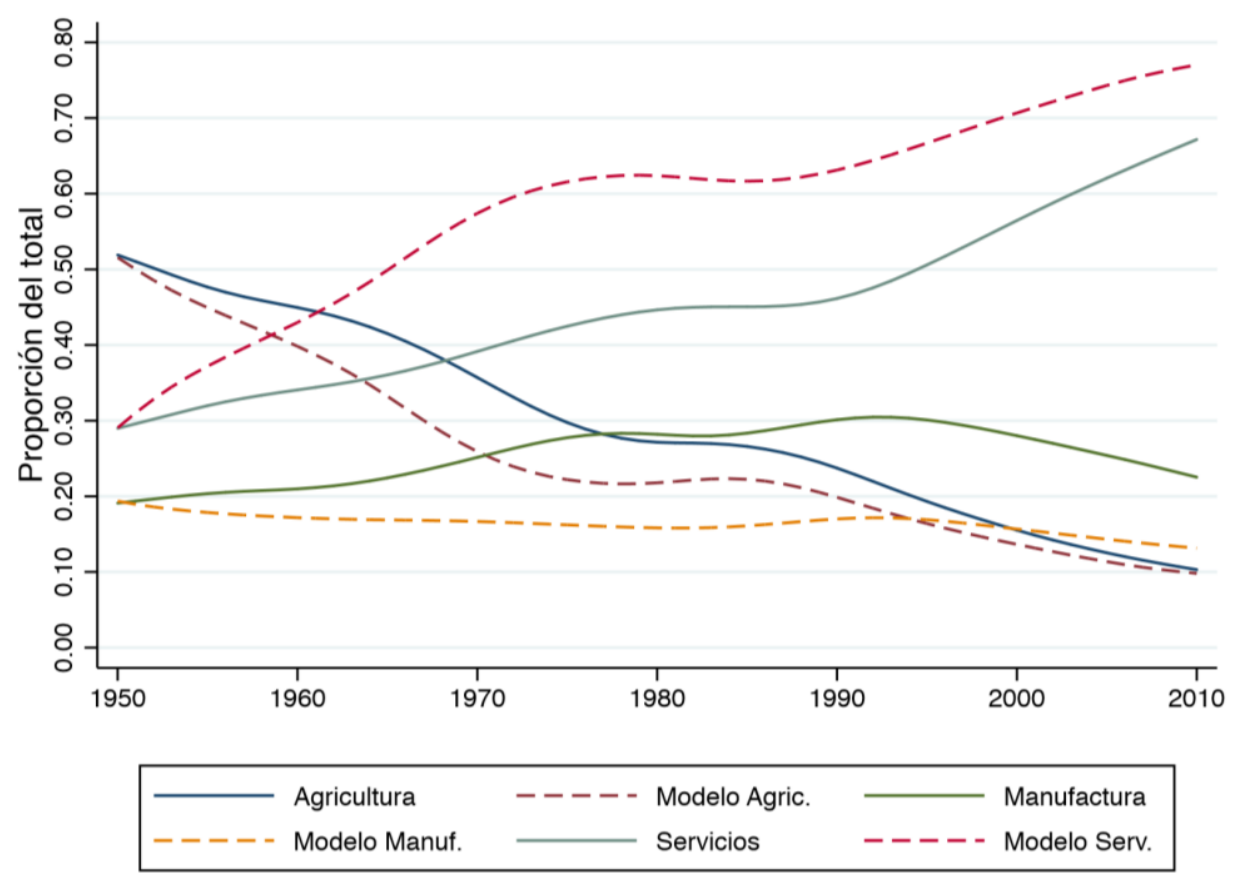

Figura 6. Modelo para Costa Rica 1950-2010. Fuente: elaboración propia.

A pesar de no realizar un ajuste, Duarte \& Restuccia (2010) discuten la imposibilidad de lograr congruencia entre el modelo y los datos de algunos países. La razón para no realizar ninguna modificación es poder comparar los resultados entre los países de la muestra. La alternativa que proponen es utilizar la metodología empleada en Duarte \& Restuccia (2007), en la que los mismos autores enfrentan un problema similar para la economía portuguesa. Se propone incorporar algún tipo de barrera que impide el movimiento de personal trabajador hacia el sector de servicios que debería ser expulsado del sector agrícola. Una alternativa es utilizar una barrera definida artificialmente $\left(\pi_{s}\right)$ para lograr que las horas trabajadas en manufactura del modelo calcen con los datos. Esta barrera se interpreta como cierto tipo de impedimentos (regulación, impuestos, etc.) que por vía de los salarios $\left(w_{s} / \pi_{s}\right)$ impiden que los grupos trabajadores puedan moverse libremente hacia el sector servicios. De esta manera se obtendría una proporción de horas para el sector manufactura que permite un mejor ajuste del modelo, tal como se aprecia en la Figura $\underline{7}$.

$$
L_{m}=\frac{L-L_{a}+\pi_{s} \bar{s} / A_{s}}{1+x}
$$




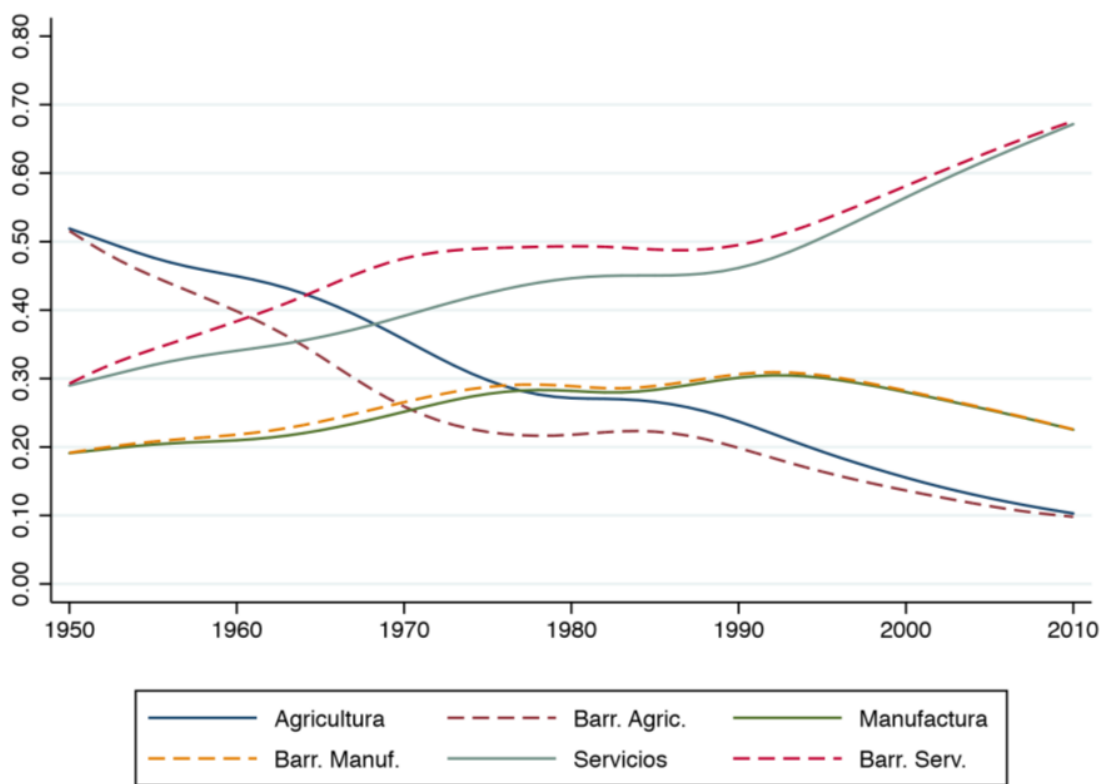

Figura 7. Modelo para Costa Rica 1950-2010: Barrera al sector servicios. Fuente: elaboración propia.

Esta barrera muestra un incremento monotónico durante la mitad del período (ver Figura 9 en el Apéndice), lo que implicaría un incremento proporcional en los costos para los grupos trabajadores de moverse entre sectores. No obstante, esta barrera deja de incrementarse (y más bien se reduce) durante las décadas más recientes, lo cual podría interpretarse como una mejoría en el funcionamiento de los mercados de trabajo en los años recientes. El ajuste con respecto a la productividad agregada es significativamente mejor para la segunda mitad del período, por lo que se tomará esta calibración con la barrera como el modelo de referencia cuando se realicen comparaciones entre países.

\section{Calibración para Costa Rica}

La estrategia alternativa es realizar el mismo ejercicio de calibración para la economía costarricense. En Costa Rica el crecimiento promedio en la productividad es de 1,46 \%, y los valores que son $\bar{a}=0,5080, \bar{s}=0,6103, b=0,2037$, y $\rho=0,1205$, y se mantiene $a=0,01$. Con respecto a estos parámetros, resulta evidente que el consumo agrícola de subsistencia es mucho mayor al de la calibración para Estados Unidos. También tiene un mayor peso en las preferencias el consumo de bienes de manufactura, determinado por el parámetro $b$.

De suma relevancia resulta que el parámetro $\rho$ para esta calibración toma un valor superior. Previamente se había mencionado que un valor más negativo implica que la reasignación de trabajo es más elástica con respecto a cambios en la productividad. En el caso de la economía 70

Alonso Alfaro Ureña / Alberto Vindas Quesada

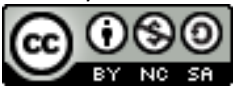

Revista Economía y Sociedad by Universidad Nacional is licensed under a CreativeCommons Reconocimiento-NoComercial- 
costarricense, el resultado es el opuesto, lo que podría explicar por qué hay una reasignación tan lenta del trabajo hacia el sector servicios. Esta calibración se puede apreciar en la Figura $\underline{8}$.

En ambas calibraciones el crecimiento de la proporción del trabajo que implica el modelo para el sector de servicios es mayor que el que se observa en los datos. El incremento en productividad de las primeras dos décadas no derivó en un crecimiento tan acelerado de la proporción de empleo en el sector servicios como lo predice el modelo. Esto ha permitido que el sector manufacturero no pierda tanto empleo como podría haberlo hecho, lo que ha contribuido a que la productividad relativa a los Estados Unidos no caiga tanto. McMillan \& Rodrik (2011) encuentran que una de las razones por las que la productividad ha caído significativamente en Latinoamérica es el incremento del tamaño de sectores en los que hay caída en la productividad, un resultado contrario a lo que se encuentra en países asiáticos.

A pesar de que en Costa Rica ha ocurrido un proceso similar, el efecto es menor del que podría haber ocurrido, si el empleo se moviera más rápido entre sectores. En términos de crecimiento de la productividad, si la reasignación de trabajo hubiese ocurrido tal como lo predice el modelo, el crecimiento promedio de la productividad en Costa Rica habría sido 1,87\%, prácticamente idéntico al que tuvo la economía estadounidense en el mismo período. Este crecimiento es significativamente mayor a $1,46 \%$ que se observa en los datos.

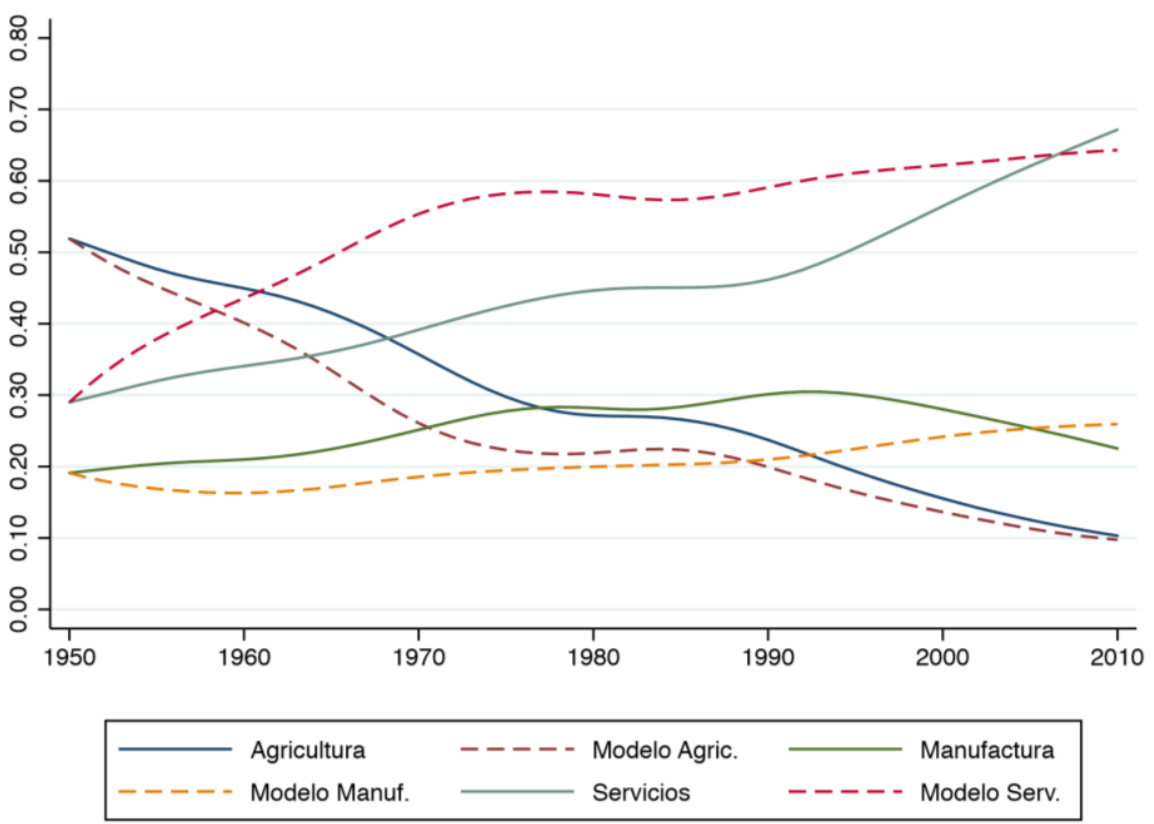

Figura 8. Calibración del modelo para Costa Rica 1950-2010. Fuente: elaboración propia. 


\section{Ejercicios contrafactuales}

En esta sección se utilizarán ambas calibraciones del modelo para realizar diferentes ejercicios contrafactuales. En el caso de la calibración para EE. UU., se presentan ejercicios que comparen la productividad de Costa Rica con respecto a la de EE. UU. La calibración para la economía costarricense permite realizar ejercicios sobre el impacto en el crecimiento en productividad de los diferentes sectores en la productividad agregada. El efecto que estos comportamientos distintos de productividad, y por ende del tamaño del empleo de cada sector, tiene sobre la productividad agregada se transmite por dos canales: el primero es directo, como se puede evidenciar en la ecuación (4.1) donde, a mayor productividad sectorial, mayor productividad agregada (manteniendo la asignación de trabajo constante). El segundo canal se da por la reasignación de trabajo entre sectores, que se comporta de acuerdo con las ecuaciones (3.7) y (3.8).

\section{Contrafactuales con la calibración para EE. UU.}

En la Tabla 1 se presentan los resultados, en donde cada columna representa un ejercicio contrafactual diferente. La primera columna presenta los datos del modelo original con la calibración para EE. UU. y los resultados para la economía costarricense. Cada columna de la 2 a la 4 representa un caso en el que la productividad de uno de los sectores crece a la misma tasa que el sector correspondiente de la economía estadounidense. La última columna presenta los resultados suponiendo que todos los sectores crecen a estas tasas.

Tabla 1

Ejercicios contrafacturales. Crecimiento de cada sector igual a EE. UU.

\begin{tabular}{lccccc}
\hline & Modelo & Agricultura & Manufactura & Servicios & Todos \\
\hline $\begin{array}{l}\text { Crecimiento en productividad (\%) } \\
\quad\end{array}$ & 3,1 & 3,7 & 3,1 & 3,1 & 3,7 \\
$\quad$ Agricultura & 2,2 & 2,2 & 1,8 & 2,2 & 1,8 \\
$\quad \begin{array}{l}\text { Manufactura } \\
\quad \text { Servicios }\end{array}$ & 0,6 & 0,6 & 0,6 & 1,3 & 1,3 \\
$\begin{array}{l}\text { Proporción de empleo 2010 (\%) } \\
\quad \text { Agricultura }\end{array}$ & 9,8 & 7,1 & 9,8 & 9,7 & 6,9 \\
$\quad$ Manufactura & 22,6 & 22,8 & 25,7 & 22,2 & 25,6 \\
$\quad$ Servicios & 67,6 & 70,1 & 64,5 & 68,1 & 67,5 \\
Productividad CR/EE. UU. & & & & & \\
$\quad$ 1950 & 0,30 & 0,30 & 0,30 & 0,30 & 0,30 \\
$\quad 2010$ & 0,37 & 0,38 & 0,34 & 0,43 & 0,42 \\
$\quad$ Crecimiento de productividad (\%) & 1,87 & 1,92 & 1,73 & 2,13 & 2,06 \\
\hline
\end{tabular}

Fuente: elaboración propia.

72

Alonso Alfaro Ureña / Alberto Vindas Quesada

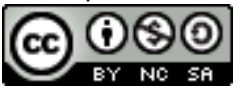

Revista Economía y Sociedad by Universidad Nacional is licensed under a CreativeCommons Reconocimiento-NoComercial- 
Los resultados indican que el impacto más significativo lo tiene el sector servicios. En particular, la productividad relativa implícita en el modelo es un 43 \% cuando se utiliza el contrafactual de crecimiento acelerado en servicios. La productividad del sector manufactura, por el contrario, ha crecido más rápidamente en Costa Rica que en EE. UU., por lo que un crecimiento similar al de este último país en ese sector, más bien habría estancado el crecimiento agregado. Es relevante notar también que los resultados del ejercicio contrafactual indican que un crecimiento de productividad más acentuado en el sector agrícola hubiera contraído más rápidamente este sector, y habría reducido la importancia relativa del empleo en este sector en una cuarta parte con respecto al modelo original.

En esta calibración resulta evidente que no hay un buen ajuste en el crecimiento de la productividad general de la economía. La economía costarricense creció en promedio 1,46 \% en ese período, mientras que el modelo predice un crecimiento de $1,87 \%$. No obstante, los ejercicios contrafactuales sirven como guía de cuál es el movimiento cualitativo que puede ocurrir a partir de cambios en los crecimientos de las productividades de cada sector. La calibración para Costa Rica resulta más precisa en el ajuste global.

\section{Contrafactuales con la calibración para Costa Rica}

La Tabla $\underline{2}$ presenta los resultados de esta calibración. La interpretación de los resultados es idéntica a la anterior, con la salvedad de que no se puede encontrar una productividad relativa a EE. UU. en estos ejercicios. En términos cualitativos los resultados de esta calibración y la anterior arrojan resultados similares. No obstante, algunos elementos resultan destacables. En primer término, un mayor crecimiento de productividad del sector servicios tiene un mayor impacto sobre el crecimiento de la productividad agregada con esta calibración.

Tabla 2

Ejercicios contrafacturales. Crecimiento de cada sector igual a EE. UU. Calibración para Costa Rica

\begin{tabular}{lccccc}
\hline & Modelo & Agricultura & Manufactura & Servicios & Todos \\
\hline Crecimiento en productividad (\%) & & & & & \\
Agricultura & 3,1 & 3,7 & 3,1 & 3,1 & 3,7 \\
Manufactura & 2,2 & 2,2 & 1,8 & 2,2 & 1,8 \\
Servicios & 0,6 & 0,6 & 0,6 & 1,3 & 1,3 \\
& & & & & \\
Proporción de empleo 2010 (\%) & 9,7 & 7,0 & 9,7 & 9,6 & 6,9 \\
Agricultura & 25,9 & 26,5 & 25,3 & 22,2 & 22,1 \\
Manufactura & & &
\end{tabular}

Fuente: elaboración propia 
Adicionalmente, la calibración para Costa Rica siempre subestima la proporción de empleo que se asigna al sector servicios para el final del período, tal como se observa en la Figura $\underline{8}$. En los ejercicios contrafactuales, especialmente cuando el crecimiento del sector servicios es mayor, este efecto es aún más evidente.

La reasignación de empleo tiene un papel preponderante en la caída de la productividad relativa del país. Si las productividades sectoriales hubiesen evolucionado tal como lo hicieron, pero si no ocurriera ninguna reasignación de empleo a partir de 1950, la productividad de la economía habría crecido un 2,46 \% en promedio. Esto significa que la productividad del país se duplicaría cada 28 años, permitiendo una convergencia mucho más rápida hacia el nivel de ingreso de los países desarrollados.

\section{Conclusiones}

Este trabajo presenta un análisis de la evolución de la productividad de la economía costarricense entre 1950 y 2010. Los datos permiten realizar un análisis desagregado que incluye los sectores agrícola, manufactura y servicios. Es posible inferir de los datos que la productividad sectorial de servicios y manufactura tuvieron incrementos muy relevantes en las décadas de los sesentas y setentas, respectivamente. Estos incrementos se contextualizan históricamente en etapas en las que los esfuerzos estatales se enfocaron en dos estrategias disímiles. Con el objetivo de lograr el desarrollo del país se aplicaron diferentes políticas económicas que tuvieron impacto en el sector de servicios primero y en el de manufactura posteriormente. No obstante, las ganancias en términos de productividad que ocurrieron en esas décadas fueron revertidas durante la década de los ochentas.

Dos elementos son de importancia alta en la caída de esta productividad. En primer término, el lento crecimiento del sector de servicios (y caída con respecto al mismo sector en la economía estadounidense) ha generado una desaceleración en el crecimiento de la productividad agregada. Adicionalmente, este sector ha ganado importancia relativa con respecto al resto de la economía, lo cual ha magnificado el efecto negativo.

Es relevante destacar que el modelo calibrado para la economía estadounidense es incapaz de replicar con exactitud el comportamiento de las asignaciones sectoriales a menos que se incluya una barrera de entrada al sector servicios. El modelo calibrado para Costa Rica permite aproximar con mayor exactitud el crecimiento de la productividad agregada, pero enfrenta problemas similares. Tal como discuten Comin, Lashkari \& Mestieri (2015), los modelos que utilizan preferencias de tipo Stone-Geary tienen dificultades para replicar la transformación estructural de economías en desarrollo. Los autores proponen incorporar funciones de utilidad GormanHanoch-Sato, que permitan tener efectos ingreso de largo plazo, así como también tendencias de precios relativos de los sectores.

74

Alonso Alfaro Ureña / Alberto Vindas Quesada

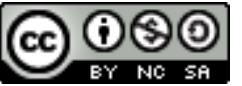

Revista Economía y Sociedad by Universidad Nacional is licensed under a CreativeCommons Reconocimiento-NoComercial- 
Existen algunas señales positivas en los datos. La productividad del sector servicios se ha incrementado levemente durante la última década, revirtiendo una tendencia negativa de alrededor de 30 años. De acuerdo con la estimación de las barreras de entrada para el modelo calibrado para EE. UU., es factible que las barreras de entrada a este sector sean cada vez menores. Adicionalmente, el crecimiento de la productividad del sector de manufactura con respecto a Estados Unidos podría contribuir a un crecimiento mayor de la productividad agregada del país.

\section{Referencias}

Abarca, A \& Ramírez, S. (2016). Estudio del crecimiento económico costarricense, 1960-2014. Observatorio del Desarrollo, Universidad de Costa Rica. Recuperado de http://www.odd.ucr.ac.cr/sites/default/files/Documents/CrecimientoEconomico/Estudio-del-Crecimiento-Economico.pdf

Heston, A., Summers, R. \& Aten, B. (2012). Penn World Table version 7.1. Center for international comparisons of production, income and prices at the University of Pennsy/vania. Recuperado de https://www.rug.nl/ggdc/productivity/pwt/pwt-releases/pwt-7.1

Comin, D., Lashkari, D., \& Mestieri, M. (2015). Structural change with long run income and price effects. Technical report. Recuperado de https://www.nber.org/papers/w21595

Duarte, M. \& Restuccia, D. (2007). The structural transformation and aggregate productivity in Portugal. Portuguese Economic Journal, 6(1), 23-46.

Duarte, M. \& Restuccia, D. (2010). The role of the structural transformation in aggregate productivity. The Quarterly Journal of Economics, 125(1), 129-173.

Feenstra, Robert C., Incklaar, R. \& Timmer, M. P. (2013). The next generation of the Penn World Table. Recuperado de www.ggdc.net/pwt.

Klenow, P. \& Rodríguez-Clare, A. (1997). The Neoclassical Revival in Growth Economics: Has It Gone Too Far? In NBER Macroeconomics Annual 1997 (Volume 12, NBER Chapters, pp. 73-114). National Bureau of Economic Research, Inc. Recuperado de https://www.nber.org/chapters/c11037

McMillan, M. S. \& Rodrik, D. (2011). Globalization, Structural Change and Productivity Growth. NBER Working Papers 17143, National Bureau of Economic Research, Inc. Recuperado de https://www.nber.org/papers/w17143.pdf

Alonso Alfaro Ureña / Alberto Vindas Quesada

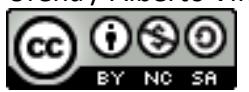

Revista Economía y Sociedad by Universidad Nacional is licensed under a CreativeCommons Reconocimiento-NoComercial- 
Ngai, L. R. \& Pissarides, C. A. (2007). Structural change in a multisector model of growth. The American Economic Review, 97(1), 429-443. Recuperado de http://personal.Ise.ac.uk/ngai/np.pdf

O'Mahony, M. \& Timmer, M. P. (2009). Output, input and productivity measures at the industry level: The EU KLEMS Database. The Economic Journal, 119(538), F374-F403. Recuperado de https://onlinelibrary.wiley.com/doi/10.1111/i.1468$\underline{0297.2009 .02280 . x}$

Organization for Economic Co-operation and Development. (2018). OECD Economic Survey of Costa Rica: Research Findings on Productivity, OECD Publishing, Paris.

Robles, E. (2018). Análisis del crecimiento de la productividad en Costa Rica, 2001-2015. Mimeo, Universidad de Costa Rica.

Mulder, N., Patiño-Pascumal, I. \& Monge-González, R. (2016). Análisis del crecimiento de la productividad en Costa Rica, 2001-2015. Estado de la Nación. Recuperado de https://estadonacion.or.cr/files/biblioteca virtual/022/Oportunidades/Mulder et a 2016.pdf

Rodríguez-Clare, A., Sáenz, M., \& Trejos, A. (2003). Análisis del crecimiento en Costa Rica. Serie Estudios Económicos y Sectoriales, RE2-03-001, IADB.

Rogerson, R. (2008). Structural transformation and the deterioration of european labor market outcomes. Journal of Political Economy, 116(2), 235-259. doi: https://doi.org/10.1086/588029

The Conference Board. (2014). Total economy database. Recuperado de http://www.conferenceboard.org/data/economydatabase/

Timmer, M., de Vries, G., \& de Vries, K. (2014). Patterns of structural change in developing countries. GGDC Research Memorandum 149. Recuperado de http://www.ggdc.net/publications/memorandum/gd149.pdf

76

Alonso Alfaro Ureña / Alberto Vindas Quesada

(c) (i) (2) (2)

Revista Economía y Sociedad by Universidad Nacional is licensed under a CreativeCommons Reconocimiento-NoComercial- 


\section{Apéndice}

\section{Descripción de bases de datos}

Para la construcción de horas trabajadas es requerido utilizar dos piezas de información: el número de personas empleadas y el número de horas trabajadas en promedio por cada una de ellas. Los datos que contiene la TED con respecto a esta última variable provienen de la Encuesta de hogares y propósitos múltiples (EHPM) que se inició en 1987. Por esta razón, no hay un dato disponible para los años previos.

El supuesto que se utiliza es que el comportamiento de las horas trabajadas para Costa Rica sigue la misma tendencia de los demás países latinoamericanos (la cual coincide con el comportamiento de los datos estadounidenses). Esta tendencia fue claramente decreciente durante los años para los cuales no hay información de Costa Rica, con un decrecimiento promedio de $0.3 \%$ para los países latinoamericanos con información disponible. Por lo tanto, se utiliza esta tendencia para reconstruir la serie de horas trabajadas promedio para los años 1950-1986. El supuesto alternativo, utilizar algún dato de horas trabajadas del período 1987-2010, sobreestimaría la productividad agregada por hora para los primeros años.

Con respecto a las horas trabajadas por sector, se utilizan las proporciones promedio (denotadas $\left.h_{i}, i \in\{a, m, s\}\right)$ para construir las horas trabajadas en cada sector para toda la extensión de la base de datos. Para Costa Rica se utilizan $h_{a}=0,8322, h_{m}=1,1051$ y $h_{s}=1,0139$. En el caso estadounidense, es posible encontrar esta proporción para todos los años deseados.

\section{Barrera hacia el sector servicios}

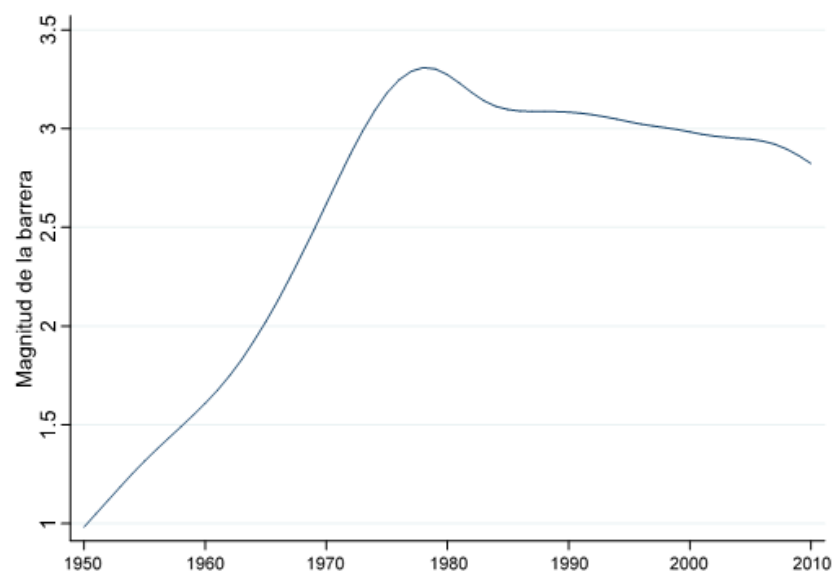

Figura 9. Costa Rica 1950-2010: barrera al sector servicios. Fuente: Elaboración propia. 